Intermolecular Coupling of Alkenes to Heterocycles via C-H Bond Activation

Kian L. Tan, Steve Park, Robert G. Bergman, ${ }^{*}$ and Jonathan A. Ellman ${ }^{*}$

Department of Chemistry, University of California and Division of Chemical Sciences, Lawrence Berkeley National Laboratory

Berkeley, CA 94720

Supporting Information

I. General Experimental Details

Page \#

II. Spectroscopic and Analytical Data

S-2

III. Selected ${ }^{1} \mathrm{H}$ NMR

S-6

S-16 


\section{Spectroscopic and Analytical Data}

\section{General Experimental.}

Unless otherwise noted, all reagents were obtained from commercial suppliers and used without further purification. All solvents were distilled under nitrogen from the following drying agents immediately prior to use: ether and tetrahydrofuran (THF) from sodium/benzophenone ketyl, and dichloromethane $\left(\mathrm{CH}_{2} \mathrm{Cl}_{2}\right)$ from calcium hydride. All reagent solutions were handled under an inert nitrogen atmosphere using syringe and cannula techniques. All reactions unless otherwise noted were carried out in flame or oven-dried glassware under an inert nitrogen atmosphere. Reaction progress was monitored with thin layer chromatography on Merck $60 F_{254} 0.25$ micron silica plates. Unless otherwise specified, extracts were dried over $\mathrm{Na}_{2} \mathrm{SO}_{4}$ and solvents were removed with a rotary evaporator. Flash column chromatography was carried out using Merck 60 230-400 mesh silica gel. All infrared (IR) spectra were recorded either on a Mattson Galaxy 3000 fourier transform spectrometer using thin film on $\mathrm{NaCl}$ plates or $\mathrm{KBr}$ pellets, or on a Nicolet Avatar 370 DTGS spectrometer using single bounce HATR on a ZnSe crystal. Only partial data are listed. ${ }^{1} \mathrm{H}$ NMR spectra were obtained on a Bruker AMX-400, AM-500 FT, or AMX-300 spectrometers. Unless otherwise noted, all NMR spectra were obtained in $\mathrm{CDCl}_{3}$; chemical shifts are reported in ppm relative to internal standard $\mathrm{CDCl}_{3}$. Coupling constants are reported in $\mathrm{Hz}$. Elemental analyses were performed by M-H-W Laboratories (Phoenix, AZ) and the University of California, Berkeley elemental analysis facility. Mass spectrometry was performed by the University of California, Berkeley mass spectrometry facility. For GC analysis yields were determined relative to internal standard, hexamethylbenzene, using a response 
factor (RF) to normalize the values. GC analysis was performed on an Agilent Technologies 6890NGC system with an Agilent Ultra 2 column $\left(140{ }^{\circ} \mathrm{C}-1 \mathrm{~min} ; 80\right.$ $\left.{ }^{\circ} \mathrm{C} / \mathrm{min}, 220{ }^{\circ} \mathrm{C}-1 \mathrm{~min} ; 80^{\circ} \mathrm{C} / \mathrm{min}, 300{ }^{\circ} \mathrm{C}-4 \mathrm{~min}\right) . \mathrm{t}_{\mathrm{R}}($ benzthiazole $)=2.854 \mathrm{~min}, \mathrm{RF}$ $=1.91 ; t_{R}($ hexamethylbenzene $)=3.663 \mathrm{~min} ; t_{R}(2$-hexylbenzthiazole $)=4.893 \mathrm{~min}, \mathrm{RF}=$ 1.05). Analytical data and synthetic protocols have been reported for the following compounds in the literature: $\left[\mathrm{RhCl}(\mathrm{coe})_{2}\right]_{2}{ }^{1}$ tert-butyldiphenylsilyl 5-hexen-1-yl ether, ${ }^{2}$ 4,4,5,5-tetramethyl-2-vinyl-[1,3] dioxolane, ${ }^{3} \mathrm{PtBu}_{2} \mathrm{Et}^{4}{ }^{4} \mathrm{PCy} \mathrm{Me}_{2},{ }^{4} \mathrm{PCy}_{2} \mathrm{Et},{ }^{4} \mathrm{PtBu} \mathrm{Bu}_{2} \mathrm{Pr},{ }^{4}$ and $\mathrm{PCy}_{2} \mathrm{tBu}{ }^{5}$.Phosphonium salts were obtained by treatment of the corresponding free base with $\mathrm{HCl}$ in ether using the procedure prescribed for the preparation of $\mathrm{PCy}_{3} \bullet \mathrm{HCl}$.

\section{General procedure coupling of heterocycles to alkenes.}

Method A. In air, to an oven dried vessel with a Kontes stopper, was added $\left[\mathrm{RhCl}(\mathrm{coe})_{2}\right]_{2}(5 \mathrm{~mol} \% \mathrm{Rh})(\mathrm{coe}=$ cis-cyclooctene $)$, tricyclohexylphosphine $(7.5 \mathrm{~mol} \%)$, lutidinium chloride $(5 \mathrm{~mol} \%)$, and the heterocycle. The vessel was placed under nitrogen. Under a positive pressure of nitrogen, THF (diluted to $0.1 \mathrm{M}$ in heterocycle) and the alkene (5 equiv) were added to the vessel. The vessel was closed with the Kontes stopper and subjected to three successive freeze pump thaw cycles. The vessel was heated for $15 \mathrm{~h}$ at $150{ }^{\circ} \mathrm{C}$. The reaction mixture was dry loaded onto $\mathrm{SiO}_{2}$ and purified by flash chromatography.

Method B. In air, to an oven dried vessel with a Kontes stopper, was added $\left[\mathrm{RhCl}(\mathrm{coe})_{2}\right]_{2}(5 \mathrm{~mol} \% \mathrm{Rh})(\mathrm{coe}=$ cis-cyclooctene $)$, tricyclohexylphosphine $(7.5 \mathrm{~mol} \%)$, and lutidinium chloride (5 mol \%). The vessel was placed under nitrogen. Under a positive pressure of nitrogen, THF, the alkene ( 5 equiv), and the heterocycle $(0.1 \mathrm{M})$ were added to the vessel. The vessel was closed with the Kontes stopper and was 
subjected to three successive freeze pump thaw cycles. The vessel was heated for 15 $\mathrm{h}$ at $150{ }^{\circ} \mathrm{C}$. The reaction mixture was dry loaded onto $\mathrm{SiO}_{2}$ and purified by flash chromatography.

Method C. In air, to an oven dried vessel with a Kontes stopper, was added $\left[\mathrm{RhCl}(\mathrm{coe})_{2}\right]_{2}(10 \mathrm{~mol} \% \mathrm{Rh})(\mathrm{coe}=$ cis-cyclooctene $)$, tricyclohexylphosphine $(15 \mathrm{~mol}$ $\%)$, lutidinium chloride ( $5 \mathrm{~mol} \%)$, and the heterocycle. The vessel was placed under nitrogen. Under a positive pressure of nitrogen, THF (diluted to $0.1 \mathrm{M}$ in heterocycle) and alkene (5 equiv) were added to the vessel. The vessel was closed with the Kontes stopper and was subjected to three successive freeze pump thaw cycles. The vessel was heated for $15 \mathrm{~h}$ at $150{ }^{\circ} \mathrm{C}$. The reaction mixture was dry loaded onto $\mathrm{SiO}_{2}$ and purified by flash chromatography.

Method D. In air, to an oven dried vessel with a Kontes stopper, was added $\left[\mathrm{RhCl}(\mathrm{coe})_{2}\right]_{2}(10 \mathrm{~mol} \% \mathrm{Rh})(\mathrm{coe}=$ cis-cyclooctene $)$, tricyclohexylphosphine $(15 \mathrm{~mol}$ $\%$ ), and lutidinium chloride (5 mol \%). The vessel was placed under nitrogen. Under a positive pressure of nitrogen, THF, the alkene ( 5 equiv), and the heterocycle $(0.1 \mathrm{M})$ were added to the vessel. The vessel was closed with the Kontes stopper and was subjected to three successive freeze pump thaw cycles. The vessel was heated for 15 $\mathrm{h}$ at $150{ }^{\circ} \mathrm{C}$. The reaction mixture was dry loaded onto $\mathrm{SiO}_{2}$ and purified by flash chromatography.

Method E. In air, to an oven dried vessel with a Kontes stopper, was added $\left[\mathrm{RhCl}(\mathrm{coe})_{2}\right]_{2}(5 \mathrm{~mol} \% \mathrm{Rh})$ (coe = cis-cyclooctene), tricyclohexylphosphonium chloride (5 mol \%), and the heterocycle. The vessel was placed under nitrogen. Under a positive pressure of nitrogen, THF (diluted to $0.1 \mathrm{M}$ in heterocycle) and the alkene ( 5 
equiv) were added to the vessel. The vessel was closed with the Kontes stopper and was subjected to three successive freeze pump thaw cycles. The vessel was heated for $15 \mathrm{~h}$ at $150{ }^{\circ} \mathrm{C}$. The reaction mixture was dry loaded onto $\mathrm{SiO}_{2}$ and purified by flash chromatography.

Method F. In air, to an oven dried vessel with a Kontes stopper, was added $\left[\mathrm{RhCl}(\mathrm{coe})_{2}\right]_{2}(10 \mathrm{~mol} \% \mathrm{Rh})(\mathrm{coe}=$ cis-cyclooctene $)$, tricyclohexylphosphonium chloride (10 mol \%), and the heterocycle. The vessel was placed under nitrogen. Under a positive pressure of nitrogen, THF (diluted to $0.1 \mathrm{M}$ in heterocycle) and the alkene ( 5 equiv) were added to the vessel. The vessel was closed with the Kontes stopper and was subjected to three successive freeze pump thaw cycles. The vessel was heated for $15 \mathrm{~h}$ at $150{ }^{\circ} \mathrm{C}$. The reaction mixture was dry loaded onto $\mathrm{SiO}_{2}$ and purified by flash chromatography.

Typical procedure for optimization screens. In a Braun glove box, a solution of benzthiazole $(0.1 \mathrm{M}),\left[\mathrm{RhCl}(\mathrm{coe})_{2}\right]_{2}(5 \mathrm{~mol} \% \mathrm{Rh}$ dimer, $10 \mathrm{~mol} \% \mathrm{Rh}), \mathrm{PCy}_{3}(10 \mathrm{~mol}$ $\%$ ), hexamethylbenzene (internal standard), and 1-hexene (5 equiv relative to benzthiazole) in THF was made. To glass vials (Kimble Article No. 60700-2) were distributed the additives $(10 \mathrm{~mol} \%)$ and the above solution $(0.5 \mathrm{~mL})$. The vessels were closed (Kontes Aritcle N0. 410119-2015 open cap with Kimble Article No. 749110-0022 valve) and were heated for $16 \mathrm{~h}$ at $75^{\circ} \mathrm{C}$ (in a Pierce No. 18935 heating block). The crude reaction mixtures were loaded onto a silica plugs, eluted with $40 \%$ EtOAc/hexanes, and analyzed by GC or ${ }^{1} \mathrm{H}$ NMR spectroscopy.

Tricyclohexylphosphonium Chloride. ${ }^{6}$ To an oven dried round bottom flask under nitrogen was added $\mathrm{PCy}_{3}(273 \mathrm{mg}, 0.975 \mathrm{mmol})$ in ether $(25 \mathrm{~mL})$. To this was 
added $4 \mathrm{~N} \mathrm{HCl}$ in dioxane $(0.5 \mathrm{~mL})$, causing immediate precipitation of a white solid. After 20 min of stirring the solid was collected by filtration and quickly washed with ether, in order to avoid exposure to moisture. After filtration the solid was dried under high vacuum with $\mathrm{P}_{2} \mathrm{O}_{5}$ as a desiccant to afford a white solid (270 mg, 87\%). ${ }^{1} \mathrm{H}$ NMR in $\mathrm{CD}_{3} \mathrm{OD}(400 \mathrm{MHz}): \delta 2.65(\mathrm{q}, 3 \mathrm{H}, J=12.0), 2.10-1.95(\mathrm{~m}, 6 \mathrm{H}), 1.90-1.85(\mathrm{~m}, 6 \mathrm{H})$, 1.80-1.70 $(\mathrm{m}, 3 \mathrm{H}), 1.70-1.55(\mathrm{~m}, 6 \mathrm{H}), 1.55-1.25(\mathrm{~m}, 9 \mathrm{H}) .{ }^{13} \mathrm{C}$ NMR in $\mathrm{CD}_{3} \mathrm{OD}(100$ $\mathrm{MHz}): \delta 27.5(\mathrm{~d}, J=3.6), 27.4(\mathrm{~d}, J=39.9), 25.8(\mathrm{~d}, J=12.9), 24.8 .{ }^{31} \mathrm{P}$ NMR in $\mathrm{CD}_{3} \mathrm{OD}$ (162 MHz): $\delta 33.8(t, J=69.9)$. . HRMS (FAB): Calc'd for $\mathrm{C}_{18} \mathrm{H}_{34} \mathrm{P}(\mathrm{M}+)$ : 281.2398. Found: 281.2396. (Note: using this procedure 1-2 equiv of $\mathrm{HCl}$ is incorporated; however, this does not appear to affect the efficiency of the reaction.)

2-(3,3-Dimethyl-butyl)-1H-benzimidazole (1). Reaction of benzimidazole (154 $\mathrm{mg}, 1.31 \mathrm{mmol})$ with neo-hexene $(810 \mu \mathrm{L}, 6.30 \mathrm{mmol})$ according to method $\mathbf{A}$ yielded the product (254 $\mathrm{mg}, 96 \%)$ as a white solid after column chromatography (10-25\% EtOAc/hexanes). Before chromatography triethylamine was added to neutralize the lutidinium chloride. The crude solution was dry loaded on to $\mathrm{SiO}_{2}$ and purified by column chromatography. M.p. $=217-218.5^{\circ} \mathrm{C} . \quad \mathrm{IR}(\mathrm{KBr}): 3057.2(\mathrm{br}, \mathrm{s}), 1415.8,1273.5$, $736.7 \mathrm{~cm}^{-1} .{ }^{1} \mathrm{H}$ NMR $\left(\mathrm{CD}_{3} \mathrm{OD}, 500 \mathrm{MHz}\right): \delta 0.99(\mathrm{~s}, 9 \mathrm{H}), 1.8-1.65(\mathrm{~m}, 2 \mathrm{H}), 2.9-2.8(\mathrm{~m}$, $2 \mathrm{H})$, 7.2-7.1 (m, 2H), 7.5-7.4 (m, 2H). ${ }^{13} \mathrm{C}$ NMR ( $\left.\mathrm{CD}_{3} \mathrm{OD}, 125 \mathrm{MHz}\right): \delta$ 156.1, 121.6, 41.9, 29.8, 28.0, 24.0 (Note: two carbons were not observed due the slow exchange of the $\mathrm{N}-\mathrm{H}$ proton, which causes these signals to be broadened). Anal. Calcd for $\mathrm{C}_{13} \mathrm{H}_{18} \mathrm{~N}_{2}$ : C, 77.18; H, 8.97; N, 13.85. Found: C, 77.04; H, 9.06; N, 13.77. El: Calc'd for $\mathrm{C}_{13} \mathrm{H}_{18} \mathrm{~N}_{2}$ $(M+):$ 202. Found: 202. 
2-(3,3-Dimethyl-butyl)-benzothiazole (2). Reaction of benzothiazole (145 $\mu \mathrm{L}$, $1.28 \mathrm{mmol})$ with neo-hexene $(810 \mu \mathrm{L}, 6.30 \mathrm{mmol})$ according to method $\mathbf{B}$ yielded the product $(272 \mathrm{mg}, 97 \%)$ as a brown/orange solid after column chromatography $(2.5 \%$ EtOAc/hexanes). Before chromatography triethylamine was added to neutralize the lutidinium chloride. The crude solution was dry loaded on to $\mathrm{SiO}_{2}$ and purified by column chromatography. IR(NaCl): $2954.7,2905.1,2864.9,758.4,728.6 \mathrm{~cm}^{-1} .{ }^{1} \mathrm{H}$ NMR (500 MHz): $\delta 1.00(\mathrm{~s}, 9 \mathrm{H}), 1.9-1.7(\mathrm{~m}, 2 \mathrm{H}), 3.2-3.0(\mathrm{~m}, 2 \mathrm{H}), 7.34$ (app. triplet, $1 \mathrm{H}, J=$ 7.5), 7.44 (app. triplet, $1 \mathrm{H}, J=7.5), 7.83(\mathrm{~d}, 1 \mathrm{H}, J=8), 7.96(\mathrm{~d}, 1 \mathrm{H}, J=8) .{ }^{13} \mathrm{C}$ NMR (125 MHz): $\delta 173.0,153.2,135.1,125.8,124.5,122.4,121.4,43.7,30.6,30.1,29.1$. Anal. Calcd for $\mathrm{C}_{13} \mathrm{H}_{17} \mathrm{NS}$ : C, 71.18; $\mathrm{H}, 7.81 ; \mathrm{N}, 6.39$. Found: $\mathrm{C}, 71.19 ; \mathrm{H}, 7.89 ; \mathrm{N}, 6.52$. 2-(3,3-Dimethyl-butyl)-1H-benzoxazole (3). Reaction of benzoxazole (154 mg, $1.27 \mathrm{mmol})$ with neo-hexene $(810 \mu \mathrm{L}, 6.30 \mathrm{mmol})$ according to method $\mathbf{A}$ yielded the product $(199 \mathrm{mg}, 77 \%)$ as a white solid after column chromatography $2.5-10 \%$ EtOAc/hexanes). Before chromatography triethylamine was added to neutralize the lutidinium chloride. The crude solution was dry loaded on to $\mathrm{SiO}_{2}$ and purified by column chromatography. IR(NaCl): 3055.5, 2955.7, 2867.1, 1572.7, 1455.3, 1242.8, $744.8 \mathrm{~cm}^{-1} .{ }^{1} \mathrm{H}$ NMR $(500 \mathrm{MHz}): \delta 0.97(\mathrm{~s}, 9 \mathrm{H}), 1.9-1.8(\mathrm{~m}, 2 \mathrm{H}), 3.0-2.8(\mathrm{~m}, 2 \mathrm{H}), 7.3-7.2$ (m, 2H), 7.5-7.4 (m, 1H), 7.7-7.6 (m, 1H). ${ }^{13} \mathrm{C}$ NMR (125 MHz): $\delta$ 167.9, 150.7, 141.3, 124.3, 124.0, 119.4, 110.1, 40.5, 30.3, 29.0, 24.3. Anal. Calcd for $\mathrm{C}_{13} \mathrm{H}_{17} \mathrm{NO}$ : C, 76.81 ; H, 8.43; N, 6.89 Found: C, 76.42; H, 8.80; N, 6.95.

2-(3,3-Dimethyl-butyl)-4, 5-dimethyl-thiazole (4). Reaction of 4, 5dimethylthiazole $(140 \mu \mathrm{L}, 1.32 \mathrm{mmol})$ with neo-hexene $(810 \mu \mathrm{L}, 6.30 \mathrm{mmol})$ according to 
method B yielded the product $(257 \mathrm{mg}, 99 \%)$ as a red/orange liquid after column chromatography ( $5 \%$ EtOAc/hexanes). Before chromatography triethylamine was added to neutralize the lutidinium chloride. The crude solution was dry loaded on to $\mathrm{SiO}_{2}$ and purified by column chromatography. IR(NaCl): 2954.0, 2866.1, 1477.8, 1365.0, $1121.5 \mathrm{~cm}^{-1} .{ }^{1} \mathrm{H}$ NMR (500 MHz): $\delta 0.93(\mathrm{~s}, 9 \mathrm{H}), 1.7-1.6(\mathrm{~m}, 2 \mathrm{H}), 2.26(\mathrm{~s}, 3 \mathrm{H}), 2.28(\mathrm{~s}$, 3H), 2.9-2.8 (m, 2H). ${ }^{13} \mathrm{C}$ NMR (125 MHz): $\delta 167.5,147.0,124.7,44.3,30.5,29.1,29.0$, 14.5, 11.2. Anal. Calcd for $\mathrm{C}_{11} \mathrm{H}_{19} \mathrm{NS}$ : C, 66.95; H, 9.70; N, 7.10 Found: $\mathrm{C}, 66.61 ; \mathrm{H}$, $9.58 ; \mathrm{N}, 7.22$.

8-(3,3-Dimethyl-butyl)-7H-purine (5). Reaction of purine (152 mg, $1.27 \mathrm{mmol})$ with neo-hexene $(810 \mu \mathrm{L}, 6.30 \mathrm{mmol}$ ) according to method $\mathbf{A}$ (with the exception that the reaction mixture was heated for only $4.5 \mathrm{~h}$ ) yielded the product (197 $\mathrm{mg}, 76 \%)$ as a white solid after column chromatography (50-100\% EtOAc/hexanes). M.p. $=179-180$ ${ }^{\circ} \mathrm{C} . \quad \mathrm{IR}(\mathrm{KBr}): 2926.5(\mathrm{br}, \mathrm{s}), 1616.7,1592.1,920.8,648.6,614,1 \mathrm{~cm}^{-1} .{ }^{1} \mathrm{H}$ NMR (CD$\left.{ }_{3} \mathrm{OD}, 500 \mathrm{MHz}\right): \delta 1.00(\mathrm{~s}, 9 \mathrm{H}), 1.8-1.7(\mathrm{~m}, 2 \mathrm{H}), 3.0-2.9(\mathrm{~m}, 2 \mathrm{H}), 8.83(\mathrm{~s}, 1 \mathrm{H}), 8.90(\mathrm{~s}$, 1H). ${ }^{13} \mathrm{C}$ NMR $(125 \mathrm{MHz}): \delta 151.3,41.3,29.9,27.9,24.6$ (Note: two carbons were not observed due the slow exchange of the $\mathrm{N}-\mathrm{H}$ proton, which causes these signals to be broadened). Anal. Calcd for $\mathrm{C}_{11} \mathrm{H}_{16} \mathrm{~N}_{4}$ : C, 64.68; H, 7.89; N, 27.43. Found: C, 64.48; $\mathrm{H}$, 7.91; N, 27.04. El: Calc'd for $\mathrm{C}_{11} \mathrm{H}_{16} \mathrm{~N}_{4}(\mathrm{M}+)$ : 204. Found: 204 .

6,8-Bis-(3, 3-Dimethyl-butyl)-7H-purine (6). Reaction of purine (152 mg, 1.27 mmol) with neo-hexene $(810 \mu \mathrm{L}, 6.30 \mathrm{mmol}$ ) according to method $\mathbf{A}$ (with the exception that the reaction mixture was only heated for $4.5 \mathrm{~h}$ ) yielded the product $(61 \mathrm{mg}, 17 \%)$ as a white solid after column chromatography (50-100\% EtOAc/hexanes). M.p. $=180-$ 
$181 .{ }^{\circ} \mathrm{C} . \quad \mathrm{IR}(\mathrm{KBr}): 2960.2(\mathrm{br}, \mathrm{s}), 1594.9,983.4,653.1 \mathrm{~cm}^{-1} .{ }^{1} \mathrm{H}$ NMR $(400 \mathrm{MHz}): \delta$ $1.00(\mathrm{~s}, 9 \mathrm{H}), 1.02(\mathrm{~s}, 9 \mathrm{H}), 1.9-1.7(\mathrm{~m}, 4 \mathrm{H}), 3.1-3.0(\mathrm{~m}, 2 \mathrm{H}), 3.3-3.1(\mathrm{~m}, 2 \mathrm{H}), 8.84(\mathrm{~s}, 1 \mathrm{H})$ ${ }^{13} \mathrm{C}$ NMR (100 MHz): $\delta 162.5,157.9,152.5,150.2,133.3,42.2,41.9,30.7,30.6,29.3$, 29.2, 28.6, 25.9. Anal. Calcd for $\mathrm{C}_{17} \mathrm{H}_{28} \mathrm{~N}_{4}: \mathrm{C}, 70.79 ; \mathrm{H}, 9.78 ; \mathrm{N}, 19.42$. Found: $\mathrm{C}$, 70.88; H, 9.86; N, 19.50.

\section{2-(3,3-Dimethyl-butyl)-1-methyl-benzimidazole (7). Reaction of 1-} methylbenzimidazole (132 mg, $1.00 \mathrm{mmol}$ ) with neo-hexene (643 $\mu \mathrm{L}, 5 \mathrm{mmol})$ according to method C yielded the product (144 $\mathrm{mg}, 67 \%)$ as a white solid after column chromatography (10-30\% EtOAc/hexanes). Before chromatography triethylamine was added to neutralize the lutidinium chloride. The crude solution was dry loaded on to $\mathrm{SiO}_{2}$ and purified by column chromatography. M.p. $=98.5-99.4{ }^{\circ} \mathrm{C} . \quad \mathrm{IR}(\mathrm{NaCl}): 2953.9$, 2864.7, $745.0 \mathrm{~cm}^{-1} .{ }^{1} \mathrm{H}$ NMR (500 MHz): $\delta 1.02(\mathrm{~s}, 9 \mathrm{H}), 1.79-1.75(\mathrm{~m}, 2 \mathrm{H}), 2.85-2.82$ (m, 2H), $3.71(\mathrm{~s}, 3 \mathrm{H}), 7.30-7.20(\mathrm{~m}, 3 \mathrm{H}), 7.72-7.70(\mathrm{~m}, 1 \mathrm{H}) .{ }^{13} \mathrm{C}$ NMR $(500 \mathrm{MHz}): \delta$ $155.8,142.5,135.8,121.9,121.7,119.0,108.7,41.4,30.4,29.6,29.1,23.0$. Anal. Calcd for $\mathrm{C}_{14} \mathrm{H}_{20} \mathrm{~N}_{2}$ : C, 77.73; H, 9.32; N, 12.95. Found: C, 77.39; H, 9.54; N, 12.63 .

8-(3,3-Dimethyl-butyl)-1, 3-dimethyl-3, 9-dihydro-purine-2, 6-dione (8). Reaction of theophyline $(100 \mathrm{mg}, 0.555 \mathrm{mmol})$ with neo-hexene $(360 \mu \mathrm{L}, 2.79 \mathrm{mmol})$ according to method $\mathbf{F}$ yielded the product $(22 \mathrm{mg}, 15 \%)$ as a white solid after column chromatography (30-50\% EtOAc/hexanes). M.p. $=233.4-234.5^{\circ} \mathrm{C} . \quad \mathrm{IR}(\mathrm{ZnSe}): 3188.0$ 2955.4, 1719.6, 1627.8 (s), $1497.6,754.3,740.2 \mathrm{~cm}^{-1} .{ }^{1} \mathrm{H}$ NMR $(500 \mathrm{MHz}): \delta 12.87(\mathrm{br}$ $\mathrm{s}, 1 \mathrm{H}), 3.63(\mathrm{~s}, 3 \mathrm{H}), 3.46(\mathrm{~s}, 3 \mathrm{H}), 2.88-2.85(\mathrm{~m}, 2 \mathrm{H}), 1.74-1.70(\mathrm{~m}, 2 \mathrm{H}), 0.98(\mathrm{~s}, 9 \mathrm{H})$. ${ }^{13} \mathrm{C}$ NMR (125 MHz): $\delta 156.9,155.7,151.5,149.5,106.4,42.2,30.4,30.2,29.0,28.3$, 
24.8. Anal. Calcd for $\mathrm{C}_{13} \mathrm{H}_{20} \mathrm{~N}_{4} \mathrm{O}_{2}$ : C, 59.07; $\mathrm{H}, 7.63 ; \mathrm{N}, 21.20$. Found: $\mathrm{C}, 58.84 ; \mathrm{H}$, $7.84 ; \mathrm{N}, 20.99$.

2-Hexyl-1H-benzimidazole (9). Reaction of benzimidazole (151 mg, $1.28 \mathrm{mmol}$ ) with 1-hexene (538 $\mathrm{mg}, 6.40 \mathrm{mmol}$ ) according to method A yielded the product (209 $\mathrm{mg}, 80 \%)$ as a white solid after column chromatography (20-50\% EtOAc/hexanes). M.p. $=134.7-135.1{ }^{\circ} \mathrm{C} . \quad \mathrm{IR}(\mathrm{KBr}): 2930.1(\mathrm{br}, \mathrm{s}), 1420.7,1027.0,750.2 \mathrm{~cm}^{-1} .{ }^{1} \mathrm{H}$ NMR $\left(\mathrm{CD}_{3} \mathrm{OD}, 500 \mathrm{MHz}\right): \delta 0.87(\mathrm{t}, 3 \mathrm{H}, \mathrm{J}=6.5), 1.4-1.2(\mathrm{~m}, 6 \mathrm{H}), 1.85-1.75(\mathrm{~m}, 2 \mathrm{H}), 2.85(\mathrm{t}$, $2 \mathrm{H}, J=7.5), 7.19-7.13(\mathrm{~m}, 2 \mathrm{H}), 7.5-7.45(\mathrm{~m}, 2 \mathrm{H}) .{ }^{13} \mathrm{C}$ NMR $\left(\mathrm{CD}_{3} \mathrm{OD}, 125 \mathrm{MHz}\right): \delta 155.5$, $121.6,31.2,28.5,28.3,27.9,22.1,12.9$ (Note: two carbons were not observed due the slow exchange of the $\mathrm{N}-\mathrm{H}$ proton, which causes these signals to be broadened). Anal. Calcd for $\mathrm{C}_{13} \mathrm{H}_{18} \mathrm{~N}_{2}$ : C, 77.18; $\mathrm{H}, 8.97 ; \mathrm{N}, 13.85$. Found: C, 77.36; $\mathrm{H}, 9.03 ; \mathrm{N}, 13.68$. El: Calc'd for $\mathrm{C}_{13} \mathrm{H}_{18} \mathrm{~N}_{2}(\mathrm{M}+)$ : 202. Found: 202 .

2-[6-(tert-Butyl-diphenyl-silanyloxy)-hexyl]-1H-benzimidazole (10). Reaction of benzimidazole $(70 \mathrm{mg}, 0.591 \mathrm{mmol})$ with tert-butyl-hex-5-enyloxy-diphenyl-silane (1 $\mathrm{g}, 2.95 \mathrm{mmol}$ ) according to method A yielded the product (207 $\mathrm{mg}, 77 \%)$ as a white solid after column chromatography (25-50\% EtOAc/hexanes). M.p. $=113.4-115{ }^{\circ} \mathrm{C}$. $\mathrm{IR}(\mathrm{KBr}): 2926.5(\mathrm{br}, \mathrm{s}), 1426.2,1273.8,1109.3,744.4,701.0 \mathrm{~cm}^{-1} .{ }^{1} \mathrm{H}$ NMR $\left(\mathrm{CD}_{3} \mathrm{OD}\right.$, $500 \mathrm{MHz}): \delta 0.99(\mathrm{~s}, 9 \mathrm{H}), 1.36-1.30(\mathrm{~m}, 2 \mathrm{H}), 1.44-1.38(\mathrm{~m}, 2 \mathrm{H}), 1.56-1.51(\mathrm{~m}, 2 \mathrm{H}), 1.82-$ $1.77(\mathrm{~m}, 2 \mathrm{H}), 2.84(\mathrm{t}, 2 \mathrm{H}, J=7.5), 3.62(\mathrm{t}, 2 \mathrm{H}, J=6.5), 7.2-7.15(\mathrm{~m}, 2 \mathrm{H}), 7.4-7.3(\mathrm{~m}, 6 \mathrm{H})$, 7.5-7.45 (m, 2H), 7.7-7.6 (m, 4H). ${ }^{13} \mathrm{C}$ NMR $\left(\mathrm{CD}_{3} \mathrm{OD}, 125 \mathrm{MHz}\right): \delta 155.4,135.2,133.6$, $129.3,127.3,121.7,63.4,32.0,28.5,28.3,27.8,25.9,25.1,18.5$ (Note: two carbons were not observed due the slow exchange of the $\mathrm{N}-\mathrm{H}$ proton, which causes these signals to be broadened). Anal. Calcd for $\mathrm{C}_{29} \mathrm{H}_{36} \mathrm{~N}_{2} \mathrm{OSi}$ : C, 76.27; $\mathrm{H}, 7.95 ; \mathrm{N}, 6.13$. 
Found: $\mathrm{C}, 76.16 ; \mathrm{H}, 8.10 ; \mathrm{N}, 6.06$. $\mathrm{FAB}$ : Calc'd for $\mathrm{C}_{29} \mathrm{H}_{36} \mathrm{~N}_{2} \mathrm{OSi}(\mathrm{M}+1)$ : 457. Found: 457.

4-(1H-Benzimidazol-2-yl)-butyric acid isobutyl ester (11). Reaction of benzimidazole $(150 \mathrm{mg}, 1.27 \mathrm{mmol})$ with isobutyl vinylacetate $(903 \mathrm{mg}, 6.35 \mathrm{mmol})$ according to method $\mathbf{C}$ (with the exception that the reaction mixture was heated for only $8 \mathrm{~h}$ ) yielded the product ( $288 \mathrm{mg}, 87 \%)$ as a white solid after column chromatography (40-75\% EtOAc/hexanes). M.p. = 96.3-100 ${ }^{\circ} \mathrm{C} . \quad \mathrm{IR}(\mathrm{KBr}): 2947.8(\mathrm{br}, \mathrm{s}), 1732.9$ 9(s), $749.3 \mathrm{~cm}^{-1} .{ }^{1} \mathrm{H}$ NMR $\left(\mathrm{CD}_{3} \mathrm{OD}, 500 \mathrm{MHz}\right): \delta 0.89(\mathrm{~d}, 6 \mathrm{H}, J=6.5), 1.90-1.82(\mathrm{~m}, 1 \mathrm{H})$, 2.15-2.10 (m, 2H), $2.41(\mathrm{t}, 2 \mathrm{H}, J=7.5), 2.93(\mathrm{t}, 2 \mathrm{H}, J=7.5), 3.80(\mathrm{~d}, 2 \mathrm{H}, J=6.5), 7.18-$ $7.16(\mathrm{~m}, 2 \mathrm{H}), 7.49-7.46(\mathrm{~m}, 2 \mathrm{H}) .{ }^{13} \mathrm{C}$ NMR $\left(\mathrm{CD}_{3} \mathrm{OD}, 125 \mathrm{MHz}\right): \delta 173.2,154.4,138.0$ (br), 121.8, 113.9 (br), 70.2, 32.8, 27.6, 27.5, 23.0, 17.9. Anal. Calcd for $\mathrm{C}_{15} \mathrm{H}_{20} \mathrm{~N}_{2} \mathrm{O}_{2}$ : C, 69.20; $\mathrm{H}, 7.74 ; \mathrm{N}, 10.76$. Found: $\mathrm{C}, 69.14 ; \mathrm{H}, 7.86 ; \mathrm{N}, 10.73$. El: Calc'd for $\mathrm{C}_{15} \mathrm{H}_{20} \mathrm{~N}_{2} \mathrm{O}_{2}$ $(\mathrm{M}+)$ : 260. Found: 260 .

2-Phenethyl-1H-benzimidazole (12). Reaction of benzimidazole (100 mg, 0.847 mmol) with styrene ( $491 \mu \mathrm{L}, 4.24 \mathrm{mmol}$ ) according to method $\mathbf{C}$ yielded the product (36 $\mathrm{mg}, 19 \%)$ as a white solid after column chromatography (25-50\% EtOAc/hexanes). The THF used in this reaction was dried over $4 \AA$ molecular sieves instead of being distilled from $\mathrm{Na}^{\circ} /$ benzophenone. GCMS analysis of the crude reaction mixture showed a linear:branched ratio of 25:1. M.p $=187.5-188.9{ }^{\circ} \mathrm{C} . \mathrm{IR}(\mathrm{KBr}): 2866.8(\mathrm{br}, \mathrm{s}), 1420.2$, 1280.1, 749.3, 696.2 $\mathrm{cm}^{-1} .{ }^{1} \mathrm{H}$ NMR ( $\left.\mathrm{CD}_{3} \mathrm{OD}, 500 \mathrm{MHz}\right): \delta 3.2-3.05(\mathrm{~m}, 4 \mathrm{H}), 7.3-7.1(\mathrm{~m}$, 7H), 7.5-7.43 (m, 2H). ${ }^{13} \mathrm{C}$ NMR $\left(\mathrm{CD}_{3} \mathrm{OD}, 125 \mathrm{MHz}\right): \delta 154.5,140.3,128.1,127.9$, $125.9,121.7,34.0,30.5$ (Note: two carbons were not observed due the slow exchange 
of the $\mathrm{N}-\mathrm{H}$ proton, which causes these signals to be broadened). HRMS (EI): Calc'd for $\mathrm{C}_{15} \mathrm{H}_{14} \mathrm{~N}_{2}(\mathrm{M}+):$ 222.1158. Found: 222.1157.

\section{2-[2-(4,4,5,5-Tetramethyl-[1,3]dioxolan-2-yl)-ethyl]-1H-benzimidazole}

Reaction of benzimidazole (150 mg, $1.27 \mathrm{mmol}$ ) with 4, 4, 5, 5-tetramethyl-2-vinyl-[1, 3]dioxolane (992 $\mathrm{mg}, 6.35 \mathrm{mmol}$ ) according to method $\mathbf{C}$ yielded the product (209 $\mathrm{mg}$, $60 \%$ ) as a white solid after column chromatography (25-75\% EtOAc/hexanes). Before chromatography triethylamine was added to neutralize the lutidinium chloride. The crude solution was dry loaded on to $\mathrm{SiO}_{2}$ and purified by column chromatography. M.p. $=202.5-204.3^{\circ} \mathrm{C} . \quad \mathrm{IR}(\mathrm{KBr}): 2976.2(\mathrm{br}, \mathrm{s}), 1547.7,1164.3,1131.4,758.9 \mathrm{~cm}^{-1} \cdot{ }^{1} \mathrm{H}$ NMR (DMSO-d 6 , $500 \mathrm{MHz}): \delta 1.12(\mathrm{~s}, 6 \mathrm{H}), 1.14(\mathrm{~s}, 6 \mathrm{H}), 2.01-1.97(\mathrm{~m}, 2 \mathrm{H}), 2.85(\mathrm{t}, 2 \mathrm{H}$,

$J=8), 5.04(\mathrm{t}, 1 \mathrm{H}, J=5), 7.09-7.08(\mathrm{~m}, 2 \mathrm{H}), 7.47-7.40(\mathrm{~m}, 2 \mathrm{H}), 12.20-12.05(\mathrm{~m}, 1 \mathrm{H}) .{ }^{13} \mathrm{C}$ NMR (DMSO-d 6 , $125 \mathrm{MHz}$ ): $\delta$ 154.8, 121.5 (br), 99.6, 81.8, 34.4, 24.5, 24.0, 22.3 (Note: two carbons were not observed due the slow exchange of the $\mathrm{N}-\mathrm{H}$ proton, which causes these signals to be broadened). Anal. Calcd for $\mathrm{C}_{16} \mathrm{H}_{22} \mathrm{~N}_{2} \mathrm{O}_{2}: \mathrm{C}, 70.04 ; \mathrm{H}, 8.08 ; \mathrm{N}$, 10.21. Found: $\mathrm{C}, 69.99 ; \mathrm{H}, 8.33 ; \mathrm{N}, 10.06$. $\mathrm{FAB}$ : Calc'd for $\mathrm{C}_{16} \mathrm{H}_{22} \mathrm{~N}_{2} \mathrm{O}_{2}(\mathrm{M}+1): 275$. Found: 275 .

2-[2-(1H-Benzimidazol-2-yl)-ethyl]isoindole-1,3-dione (14). Benzimidazole (50 mg, $0.42 \mathrm{mmol}$ ) was treated with $\mathrm{N}$-vinylphthalimide (367 mg, $2.12 \mathrm{mmol}$ ) according to method F. However, the work-up was modified to remove excess alkene. Upon completion of the reaction the mixture was diluted with EtOAc $(40 \mathrm{~mL})$. The organic layer was extracted with $1 \mathrm{~N} \mathrm{HCl}(3 \times 15 \mathrm{~mL})$. The aqueous layer was basified with $\mathrm{K}_{2} \mathrm{CO}_{3}$ and extracted with $\mathrm{CH}_{2} \mathrm{Cl}_{2}(3 \times 15 \mathrm{~mL})$, and the $\mathrm{CH}_{2} \mathrm{Cl}_{2}$ layers were combined, concentrated, and purified by $\mathrm{SiO}_{2}$ column chromatography (50-75\% EtOAc/hexanes). 
The product was isolated as a white solid $(78 \mathrm{mg}, 63 \%)$. M.p. $=192.7-194.3{ }^{\circ} \mathrm{C} . \quad$ IR (ZnSe): $3322.1(\mathrm{br}), 2946.1,1769.6,1705.6(\mathrm{~s}), 1396.2,714.3 \mathrm{~cm}^{-1} .{ }^{1} \mathrm{H}$ NMR $(500$ $\left.\mathrm{MHz}, \mathrm{CD}_{3} \mathrm{OD}\right) \delta$ 7.73-7.68 (m, 4H), 7.43-7.41 (m, 2H), 7.14-7.12 (m, 2H), $4.08(\mathrm{t}, 2 \mathrm{H}, J$ $=6.9), 3.21(\mathrm{t}, 2 \mathrm{H}, J=6.9) .{ }^{13} \mathrm{C} \operatorname{NMR}\left(125 \mathrm{MHz}, \mathrm{CD}_{3} \mathrm{OD}\right): \delta 169.3,153.2,139.6(\mathrm{br})$. 135.2, 133.2, 124.1, 123.3, 115.5 (br), 37.5, 29.0. HRMS (FAB): Calc'd for $\mathrm{C}_{17} \mathrm{H}_{14} \mathrm{~N}_{3} \mathrm{O}_{2}$ $(M+1): 292.1086$. Found: 292.1085 .

3-(4,5-Dimethyl-thiazol-2-yl)-propionic acid tert-butyl ester (15). Reaction of 4, 5-dimethylthiazole (97\% pure, $135 \mu \mathrm{L}, 1.24 \mathrm{mmol}$ ) with tert-butyl acrylate (930 $\mu \mathrm{L}$, $6.35 \mathrm{mmol}$ ) according to method $\mathbf{D}$ yielded the product $(278 \mathrm{mg}, 93 \%)$ as a red-orange liquid after column chromatography (5-10\% EtOAc/hexanes). Before chromatography triethylamine was added to neutralize the lutidinium chloride. The crude solution was dry loaded on to $\mathrm{SiO}_{2}$ and purified by column chromatography. $\operatorname{IR}(\mathrm{NaCl}): 2978.9$, 2924.5, 1729.1, 1366.88, $1154.65 \mathrm{~cm}^{-1} .{ }^{1} \mathrm{H}$ NMR (500 MHz): $\delta 1.42(\mathrm{~s}, 9 \mathrm{H}), 2.25(\mathrm{~s}$, $3 \mathrm{H}), 2.29(\mathrm{~s}, 3 \mathrm{H}), 2.66(\mathrm{t}, 2 \mathrm{H}, J=7.5), 3.14(\mathrm{t}, 2 \mathrm{H}, J=7.5) .{ }^{13} \mathrm{C}$ NMR $(125 \mathrm{MHz}): \delta 171.4$, 164.3, 147.3, 125.2, 80.6, 35.1, 28.5, 28.0, 14.5, 11.2. Anal. Calcd for $\mathrm{C}_{12} \mathrm{H}_{19} \mathrm{NO}_{2} \mathrm{~S}: \mathrm{C}$, 59.72; H, 7.93; N, 5.80. Found: C, 59.75; H, 7.79; N, 6.00.

3-(4,5-Dimethyl-thiazol-2-yl)-propionitrile (16). Reaction of $4, \quad 5$ dimethylthiazole ( $97 \%$ pure, $135 \mu \mathrm{L}, 1.24 \mathrm{mmol})$ with acrylonitrile $(418 \mu \mathrm{L}, 6.35 \mathrm{mmol})$ according to method $\mathbf{D}$ yielded the product (134 $\mathrm{mg}, 65 \%$ linear product) as a yellow liquid after column chromatography (10-30\% EtOAc/hexanes). GCMS analysis of the crude reaction mixture showed a linear:branched ratio of 4.3:1. $I R(N a C l): 2922.8$, 2864.8, 2249.3, 1439.7, $1134.8 \mathrm{~cm}^{-1} .{ }^{1} \mathrm{H}$ NMR (400 MHz): $\delta 2.27$ (s, 3H), $2.30(\mathrm{~s}, 3 \mathrm{H})$, 
$2.80(\mathrm{t}, 2 \mathrm{H}, J=7.6), 3.19(\mathrm{t}, 2 \mathrm{H}, J=7.6) .{ }^{13} \mathrm{C}$ NMR $(100 \mathrm{MHz}): \delta 160.6,148.2,126.5$, 118.6, 28.9, 17.3, 14.6, 11.2. Anal. Calcd for $\mathrm{C}_{8} \mathrm{H}_{10} \mathrm{~N}_{2} \mathrm{~S}$ : C, 57.80; $\mathrm{H}, 6.06 ; \mathrm{N}, 16.85$. Found: C, 57.81; $\mathrm{H}, 6.05 ; \mathrm{N}, 17.04$.

2-(3,3-Dimethyl-butyl)-6-methoxy-1H-benzimidazole (17). Reaction of 5methoxybenzimidazole $(100 \mathrm{mg}, 0.675 \mathrm{mmol})$ with neo-hexene $(283 \mu \mathrm{L}, 2.20 \mathrm{mmol})$ according to method $\mathrm{E}$ yielded the product (148 $\mathrm{mg}, 94 \%$ ) as a yellow solid after column chromatography (25-50\% EtOAc/hexanes). M.p. $=94.6-96{ }^{\circ} \mathrm{C} \mathrm{IR}(\mathrm{ZnSe}): 3300-2600$ (br), $1156.6 \mathrm{~cm}^{-1} .{ }^{1} \mathrm{H}$ NMR (500 MHz): $\delta 9.30(\mathrm{~s}, \mathrm{br}, 1 \mathrm{H}), 7.41(\mathrm{~d}, 1 \mathrm{H}, J=8.7), 7.01(\mathrm{~d}$, $1 \mathrm{H}, J=2.2), 6.83(\mathrm{dd}, 1 \mathrm{H}, J=2.3,8.7), 3.79(\mathrm{~s}, 3 \mathrm{H}), 2.88-2.85(\mathrm{~m}, 2 \mathrm{H}), 1.77-1.73(\mathrm{~m}$, 2H), 0.84 (s, 9H). ${ }^{13} \mathrm{C}$ NMR (125 MHz): $\delta 156.0,155.8,138.7(\mathrm{br}), 133.4(\mathrm{br}), 115.2(\mathrm{br})$, 111.2, 97.5 (br), 55.8, 42.2, 30.3, 29.0, 24.8. Anal. Calcd for $\mathrm{C}_{14} \mathrm{H}_{20} \mathrm{~N}_{2} \mathrm{O}: \mathrm{C}, 72.38 ; \mathrm{H}$, 8.68; N, 12.06. Found: C, 72.09; H, 8.96; N, 11.76.

2-(3,3-Dimethyl-butyl)-3H-benzimidazole-5-carboxylic acid (18). Reaction of 5-benzimidazole carboxylic acid $(100 \mathrm{mg}, 0.617 \mathrm{mmol})$ with neo-hexene $(397 \mu \mathrm{L}, 3.08$ $\mathrm{mmol}$ ) according to method $\mathrm{E}$ yielded the product (101 $\mathrm{mg}, 66 \%$ ) as a pale yellow solid after column chromatography (5-10\% $\left.\mathrm{MeOH} / \mathrm{CH}_{2} \mathrm{Cl}_{2}\right)$. IR(ZnSe): $3300-2600$ (br), $1683.1(\mathrm{w}), 1365.7,776.5 \mathrm{~cm}^{-1} .{ }^{1} \mathrm{H}$ NMR (500 MHz, $\left.\mathrm{CD}_{3} \mathrm{OD}\right) ; \delta 8.19(\mathrm{~s}, 1 \mathrm{H}), 7.90(\mathrm{~d}$, $1 \mathrm{H}, J=8.4), 7.48(\mathrm{~d}, 1 \mathrm{H}, J=8.4), 2.87-2.84(\mathrm{~m}, 2 \mathrm{H}), 1.72-1.69(\mathrm{~m}, 2 \mathrm{H}), 0.95(\mathrm{~s}, 9 \mathrm{H})$. ${ }^{13} \mathrm{C}$ NMR $\left(125 \mathrm{MHz}, \mathrm{CD}_{3} \mathrm{OD}\right): \delta 171.2,160.2,142.5,139.2,126.8,125.3,118.0,114.8$, 43.3, 31.4, 29.6, 25.7. HRMS (FAB): Calc'd for $\mathrm{C}_{14} \mathrm{H}_{19} \mathrm{~N}_{2} \mathrm{O}_{2}(\mathrm{M}+1)$ : 247.1449. Found: 247.1447. 


\section{2-(3,3-Dimethyl-butyl)-3H-benzimidazole-5-carboxylic acid methyl ester (19).}

Reaction of 5-benzimidazole carboxylic acid methyl ester (100 mg, $0.568 \mathrm{mmol}$ ) with neo-hexene ( $365 \mu \mathrm{L}, 2.84 \mathrm{mmol}$ ) according to method $\mathrm{E}$ yielded the product (132 $\mathrm{mg}$, $89 \%$ ) as a yellow oil after column chromatography (EtOAc/hexanes). IR(ZnSe): $3300-$ 2600 (br), 1715.8 (s), 1285.4, $771.9 \mathrm{~cm}^{-1} . \quad{ }^{1} \mathrm{H}$ NMR (500 MHz): $\delta$ 10.0-9.0 (br, s, $\left.1 \mathrm{H}\right)$, $8.30(\mathrm{~s}, 1 \mathrm{H}), 7.93(\mathrm{~d}, 1 \mathrm{H}, J=8.5), 7.54(\mathrm{~d}, 1 \mathrm{H}, J=8.5), 3.89(\mathrm{~s}, 3 \mathrm{H}), 2.96-2.92(\mathrm{~m}, 2 \mathrm{H})$, 1.79-1.75 (m, 2H), $0.79(\mathrm{~s}, 9 \mathrm{H}) .{ }^{13} \mathrm{C}$ NMR (125 MHz): $\delta$ 167.9, $159.0(\mathrm{br}), 141.9(\mathrm{br})$, 138.1 (br), 123.9, 123.8, 116.8 (br), 114.1 (br), 52.1, 42.2, 30.3, 28.8, 24.9. HRMS (FAB): Calc'd for $\mathrm{C}_{15} \mathrm{H}_{20} \mathrm{~N}_{2} \mathrm{O}_{2}(\mathrm{M}+1)$ : 261.1603. Found: 261.1603 .

6-Chloro-2-(3,3-dimethyl-butyl)-1H-benzimidazole (20). Reaction of 5chlorobenzimidazole $(100 \mathrm{mg}, 0.655 \mathrm{mmol})$ with neo-hexene $(421 \mu \mathrm{L}, 3.28 \mathrm{mmol})$ according to method $\mathrm{E}$ yielded the product (143 $\mathrm{mg}, 93 \%$ ) as a yellow solid after column chromatography $\left(25 \%\right.$ EtOAc/hexanes). M.p. $=159-163{ }^{\circ} \mathrm{C} . \quad$ IR $(\mathrm{ZnSe}): 3300-2600$ (br), 1410.7, $801.3 \mathrm{~cm}^{-1} .{ }^{1} \mathrm{H}$ NMR (500 MHz): $\delta$ 13.0-12.5 (br s, 1H), 7.55 (s, 1H), 7.46 (d, $1 \mathrm{H}, J=8.5), 7.20(\mathrm{~d}, 1 \mathrm{H}, J=8.5), 2.97-2.94(\mathrm{~m}, 2 \mathrm{H}), 1.81-1.78(\mathrm{~m}, 2 \mathrm{H}), 0.82(\mathrm{~s}, 9 \mathrm{H})$. ${ }^{13} \mathrm{C}$ NMR (125 MHz): 157.8, 139.3, 137.0, 127.7, 122.6, 115.1, 114.5, 42.5, 30.3, 28.9, 24.9. Anal. Calcd. for $\mathrm{C}_{13} \mathrm{H}_{17} \mathrm{CIN}_{2}$ : $\mathrm{C}, 65.95 ; \mathrm{H}, 7.24 ; \mathrm{N}, 11.83$. Found: $\mathrm{C}, 65.75 ; \mathrm{H}$, $7.31 ; \mathrm{N}, 11.88$.

2-(3,3-Dimethyl-butyl)-6-trifluoromethyl-1H-benzimidazole (21). Reaction of 5-trifluoromethylbenzimidazole $(100 \mathrm{mg}, 0.543 \mathrm{mmol})$ with neo-hexene $(350 \mu \mathrm{L}, 2.72$ $\mathrm{mmol}$ ) according to method E yielded the product (139 $\mathrm{mg}, 95 \%)$ as a yellow solid after column chromatography (25\% EtOAc/hexanes). M.p. $=174-179{ }^{\circ} \mathrm{C} . \quad$ IR $(\mathrm{ATIR}): 3300-$ 
2600, 2924.5, 1327.6, 1112.6, $812.9 \mathrm{~cm}^{-1} .{ }^{1} \mathrm{H}$ NMR $\left(500 \mathrm{MHz}, \mathrm{CD}_{3} \mathrm{OD}\right): \delta 7.77(\mathrm{~s}, 1 \mathrm{H})$, $7.58(\mathrm{~d}, 1 \mathrm{H} J=8.4), 7.42(\mathrm{~d}, 1 \mathrm{H}, J=7.5), 2.88-2.85(\mathrm{~m}, 2 \mathrm{H}), 173-1.70(\mathrm{~m}, 2 \mathrm{H}), 0.96(\mathrm{~s}$, 9H). ${ }^{13} \mathrm{C}$ NMR (125 MHz, $\mathrm{CD}_{3} \mathrm{OD}$ ): $\delta$ 160.4, 141.2 (br), 139.7 (br), 126.4 (q, $J=271$ ), 125.3 (q, $J=32$ ), 119 (q, $J=3.6), 115.4(b r), 113.3(b r), 43.2,31.2,29.4,25.6 .{ }^{19} \mathrm{~F}$ NMR (376 MHz): $\delta-61.2$. Anal. Calcd. for $\mathrm{C}_{14} \mathrm{H}_{17} \mathrm{~N}_{2} \mathrm{~F}_{3}: \mathrm{C}, 62.21 ; \mathrm{H}, 6.34 ; \mathrm{N}, 10.36$. Found: C, 62.17; H, 6.48; N, 9.97.

2-Hexylbenzthiazole (22). ${ }^{7}$ Reaction of benzthiazole $(55 \mathrm{mg}, 0.407 \mathrm{mmol})$ with 1-hexene ( $231 \mu \mathrm{L}, 1.85 \mathrm{mmol}$ ) according to method E yielded the product (46 mg, 52\%) as a red oil after column chromatography (2.5\% EtOAc/hexanes). $\operatorname{IR}(\mathrm{ZnSe}): 2926.5$, 2856.1, 1520.0, 757.2, $727.7 \mathrm{~cm}^{-1} .{ }^{1} \mathrm{H}$ NMR (500 MHz): $\delta 7.96(\mathrm{~d}, 1 \mathrm{H}, J=8.1), 7.82(\mathrm{~d}$, $1 \mathrm{H}, J=7.9), 7.43(\mathrm{t}, 1 \mathrm{H}, J=7.4), 7.32(\mathrm{t}, 1 \mathrm{H}, J=7.5), 3.10(\mathrm{t}, 2 \mathrm{H}, J=7.7), 1.88-1.83$ $(\mathrm{m}, 2 \mathrm{H}), 1.45-1.43(\mathrm{~m}, 2 \mathrm{H}), 1.33-1.25(\mathrm{~m}, 4 \mathrm{H}), 0.90-0.87(\mathrm{~m}, 3 \mathrm{H}) .{ }^{13} \mathrm{C}$ NMR $(125 \mathrm{MHz})$ : $\delta 172.4,153.2,135.1,125.8,124.5,122.4,121.4,34.3,31.4,29.7,28.8,22.5,14.0$.

\section{Selected Spectra}

${ }^{1}$ HNMR of 12 


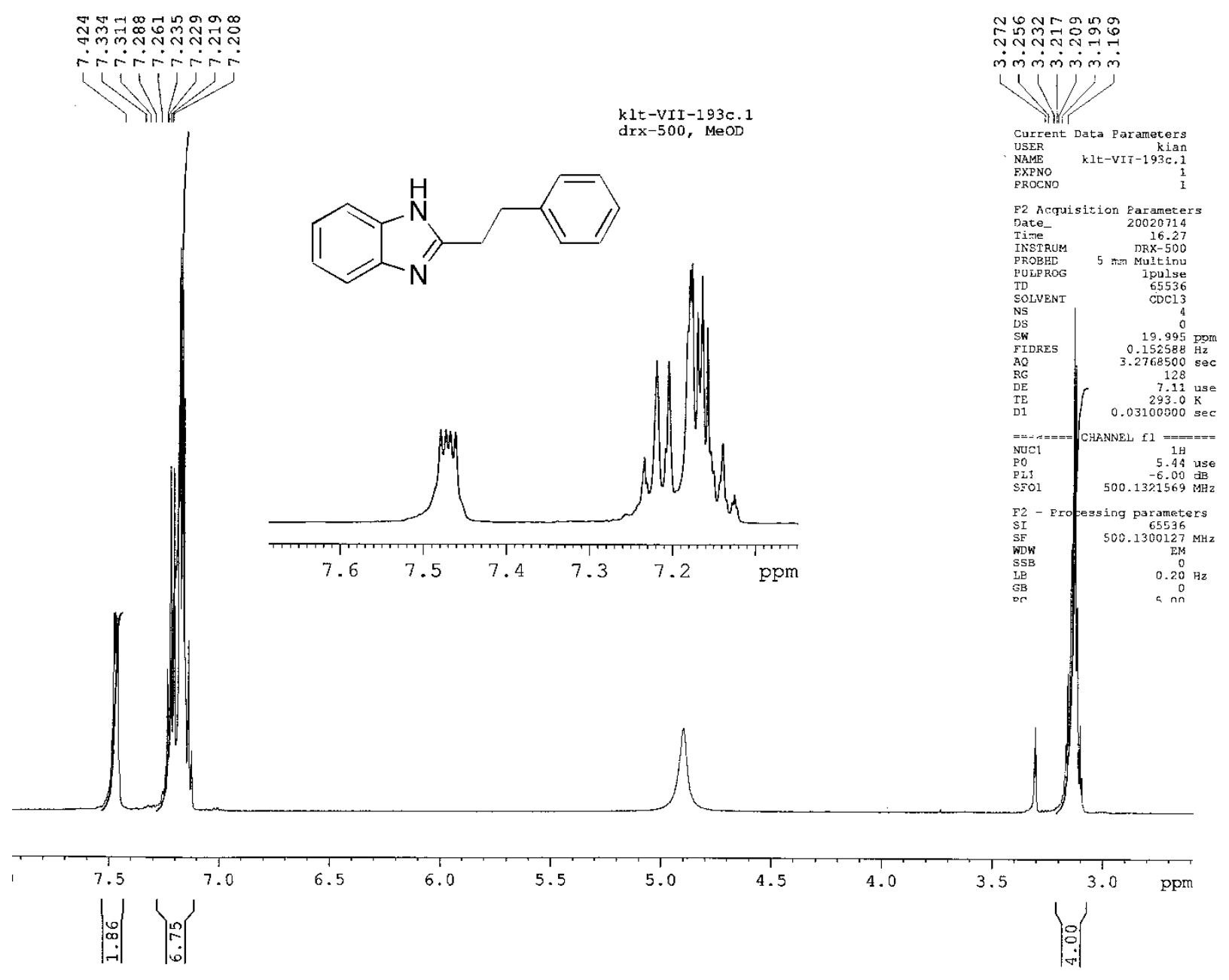

${ }^{1}$ HNMR of 14 

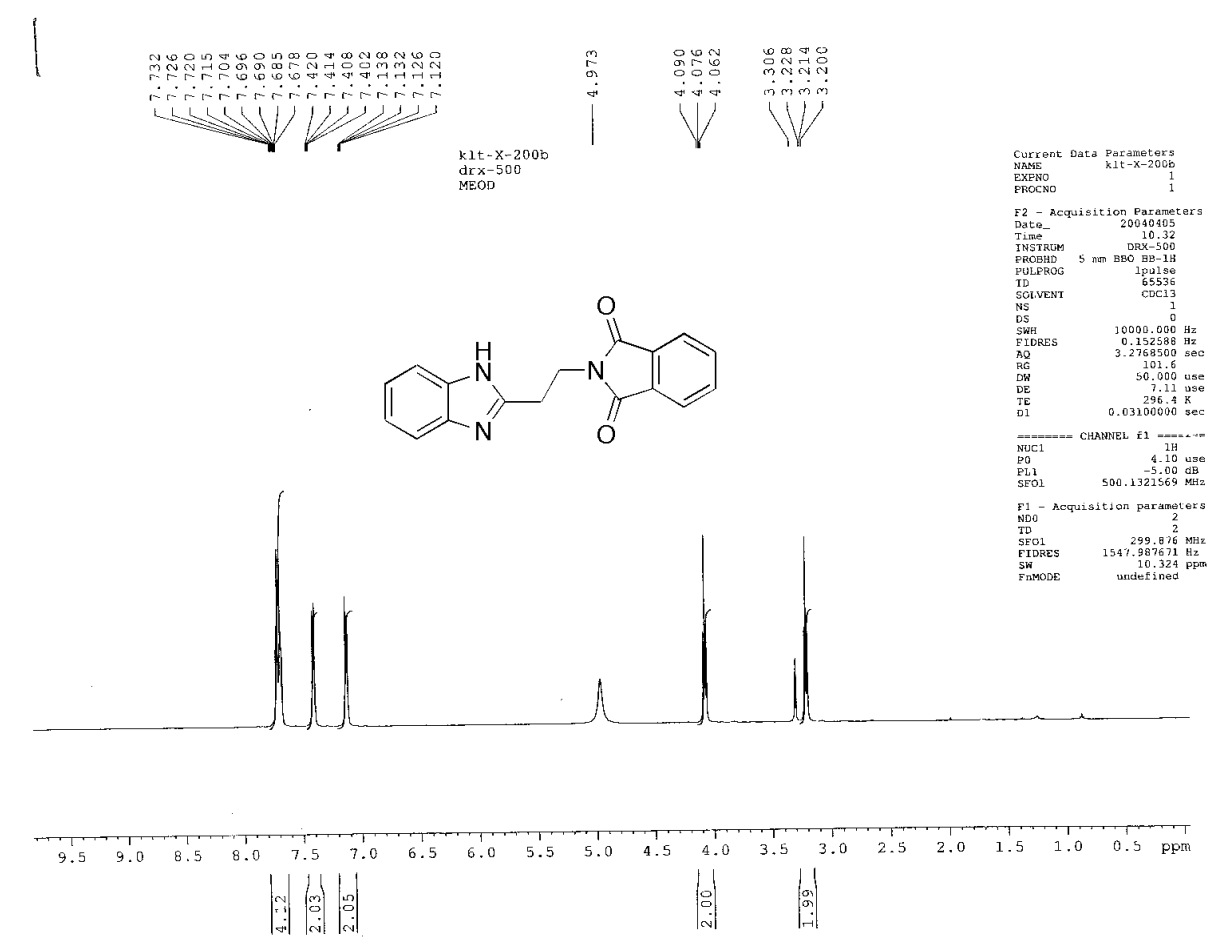

\section{${ }^{1}$ HNMR of 18}

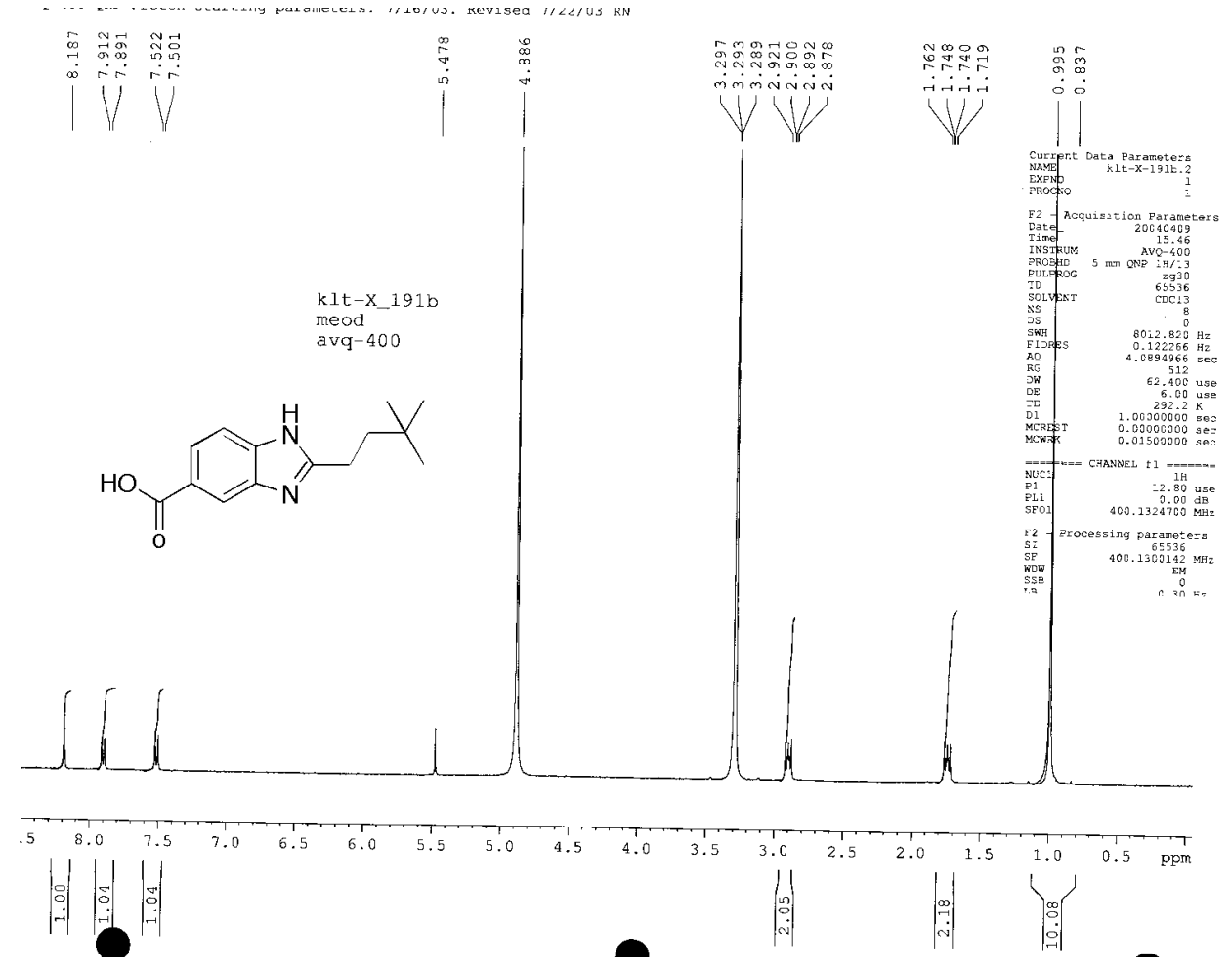

${ }^{1}$ HNMR of 19 


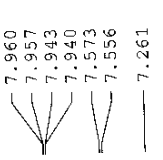

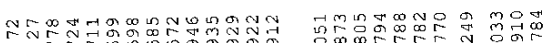

कo

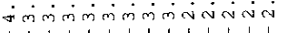

hlWh/

Vilif|

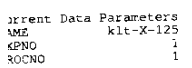

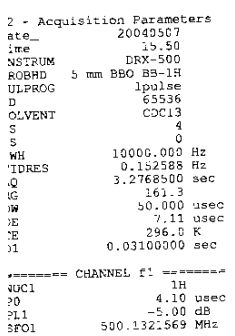

F1 - Acquisition paraneters

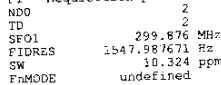
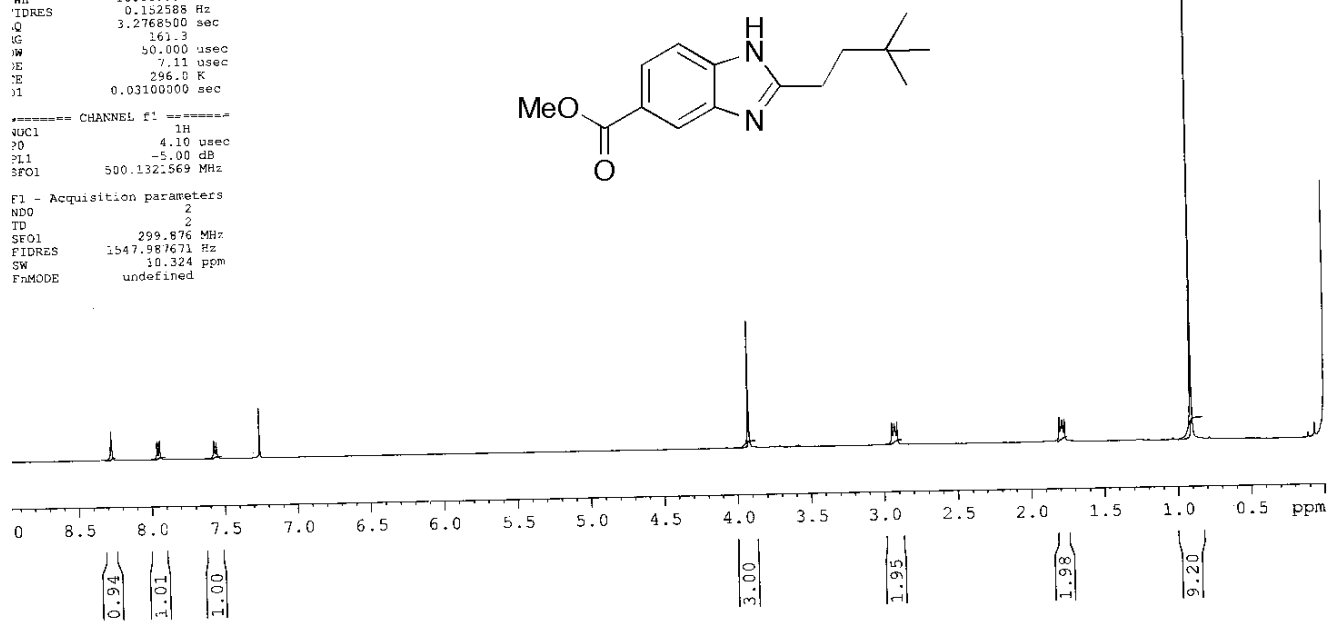

\section{${ }^{1} \mathrm{HNMR}$ of $\mathrm{HCIPCy}_{3}$}
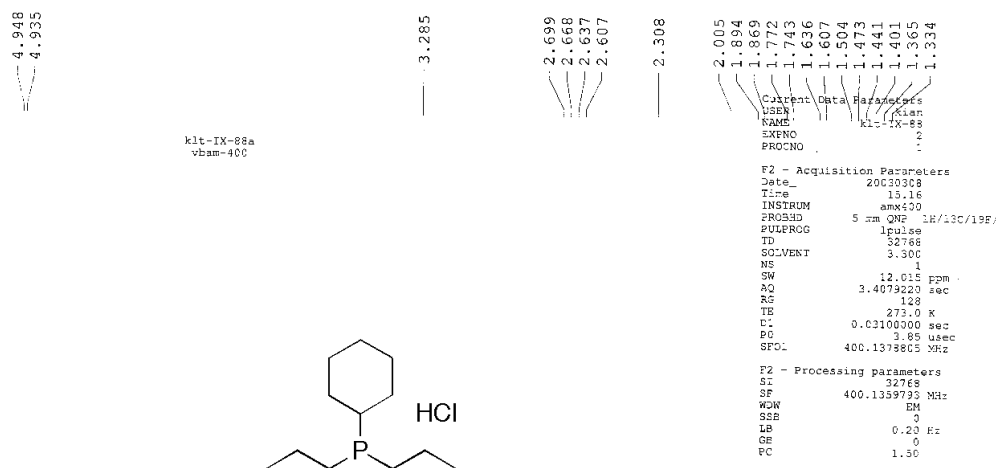<smiles>ClC1CCCCC1P(C1CCCCC1)C1CCCCC1</smiles>

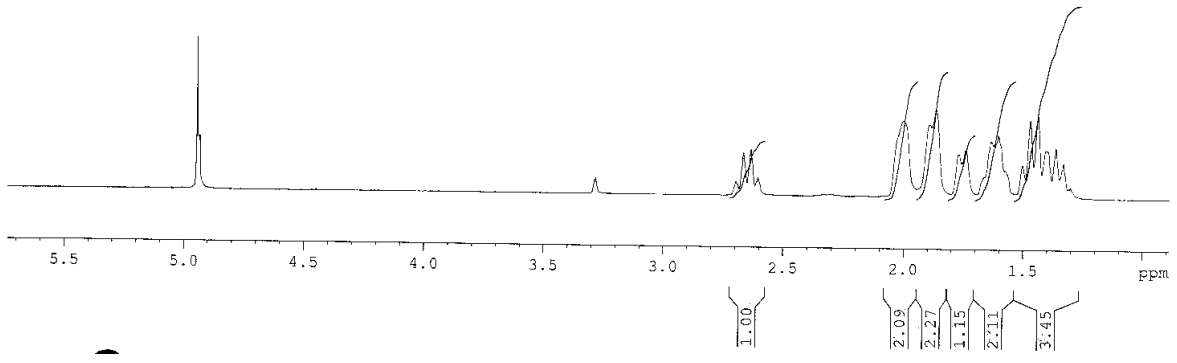


${ }^{1}$ Ent, A.; Onderdelinden, A. L. Inorg. Syth. 1973, 14, 92-93.

${ }^{2}$ Fujiwara, K.; Amano, A.; Tokiwano, T.; Murai, A. Tetrahedron 2000, 56, 1065-1080. ${ }^{3}$ Deagostino, A.; Maddaluno, J.; Mella, M.; Prandi, C.; Venturello, P. J. Chem. Soc., Perkin Trans. 1 1998, 881-888.

${ }^{4}$ Netherton, M. R.; Fu, G. C. Angew. Chem., Int. Edit. Engl. 2002, 41, 3910-3912. 5 Jan, D.; Delaude, L.; Simal, F.; Demonceau, A.; Noels, A. F. J. Organomet. Chem. 2000, 606, 55-64.

${ }^{6}$ Note that a between 1-2 equiv of $\mathrm{HCl}$ was incorporated in this procedure; however, this did not effect the reactivity of the catalyst.

${ }^{7}$ Boger, D J. Org. Chem. 1978, 11, 2296-2297. 